\title{
Obstrução intestinal associada a tricobezoares de grande volume - um relato de caso
}

\author{
Bowel obstruction associated with large volume trichobezoars - a case report \\ Obstrucción intestinal asociada a tricobezoares de gran volumen - un relato de caso
}

Raphaela Silva de Souza1, Brígida Thaine Fernandes Cabral'3 , Juan Eduardo Rios Rodriguez ${ }^{3}$, Lúcia Tatiana Filgueiras de Souza ${ }^{4}$, Thais Dourado de Andrade ${ }^{5}$, Izabela Augusta De Oliveira Medeiros ${ }^{2}$.

\section{RESUMO}

Objetivo: Relatar um caso de obstrução intestinal causada por massa formada a partir da ingesta de pelos Relato do caso: Trata- se de paciente do sexo feminino, 15 anos, procura o serviço de Pronto Socorro com relato de constipação intestinal há 15 dias, sem comorbidades conhecidas até o momento. Após lavagem intestinal com clister glicerinado, foi avaliada com melhora e alta no mesmo dia. Após 24 horas, evoluiu com vômitos fecalóides, distensão abdominal e febre não aferida. Após estabilização hemodinâmica, foi realizada tomografia computadorizada de abdômen, evidenciando sinais de obstrução intestinal sem fator obstrutivo evidente. Foi indicada laparotomia exploradora para melhor avaliação e tratamento do caso. Discussão: Tricobezoares são causas raras na população em geral, mas em populações específicas, como adolescentes do sexo feminino por exemplo, tendem a ser mais comuns. Não obstante, apenas o tratamento cirúrgico não é suficiente no tratamento, devendo a mesma ser encaminhada aos serviços de assistência social, psicologia e psiquiatria, para evitar novos casos e complicações.

Palavras-Chave: Abdome, Cirurgia, Obstrução Intestinal.

\begin{abstract}
Objetive: Report a case of intestinal obstruction caused by mass formed from the ingestion of pellets. Case report: This is a female patient, 15 years old, seeking the emergency room service for 15 days without constipation. comorbidities known to date. After intestinal lavage with glycerinated clister, it was evaluated with improvement and discharge on the same day. After 24 hours, he evolved with fecal vomiting, abdominal distension and unmeasured fever. After hemodynamic stabilization, computed tomography of the abdomen was performed, evidencing signs of intestinal obstruction without obvious obstructive factor. Exploratory laparotomy was indicated for better evaluation and treatment of the case. Discussion: Trichobezoars are rare causes in the general population, but in specific populations, such as female adolescents, for example, tend to be more common. Nevertheless, only the surgical treatment is not enough in the treatment, and it should be referred to the social services, psychology and psychiatry, to avoid new cases and complications.
\end{abstract}

Key words: Abdome, Surgery, Intestinal Obstruction.

\footnotetext{
${ }^{1}$ Hospital Universitário Getúlio Vargas (HUGV), Manaus-AM. * E-mail: kadmiel_cec@hotmail.com

2 Instituto de Cirurgia do Estado do Amazonas (ICEA)

3 Universidade Federal do Amazonas (UFAM)

${ }^{4}$ Universidade do Estado do Amazonas (UEA)

${ }^{5}$ Universidade Nilton Lins (UNL)
} 


\section{RESUMEN}

Objetivo: Informar de un caso de obstrucción intestinal causada por masa formada a partir de la ingesta del mismo. Relato del caso: Se trata de paciente del sexo femenino, 15 años, busca el servicio de Pronto Socorro con relato de constipación intestinal hace 15 días, sin las comorbilidades conocidas hasta el momento. Después del lavado intestinal con clister glicerinado, fue evaluada con mejoría y alta en el mismo día. Después de 24 horas, evolucionó con vómitos fecalóides, distensión abdominal y fiebre no aferida. Después de la estabilización hemodinámica, se realizó tomografía computarizada de abdomen, evidenciando signos de obstrucción intestinal sin factor obstructivo evidente. Se ha indicado laparotomía exploradora para una mejor evaluación y tratamiento del caso. Discusión: Tricobezoares son causas raras en la población en general, pero en poblaciones específicas, como las adolescentes, por ejemplo, tienden a ser más comunes. No obstante, sólo el tratamiento quirúrgico no es suficiente en el tratamiento, debiendo la misma ser encaminada a los servicios de asistencia social, psicología y psiquiatría, para evitar nuevos casos y complicaciones.

Palabras clave: Abdomen; Cirurgia; Obstruccion Intestinal.

\section{INTRODUÇÃO}

O termo "bezoar" tem origem persa e é definido por massas endurecidas, com diversas etiologias, e que podem ser formados em diferentes alturas do trato gastrointestinal. Os primeiros relatos descritos acerca de tricobezoares humanos datam do fim do século XVIII, não obstante muitos casos já haviam sido citados em outras espécies anteriormente. À vista disso, estudos sobre os mesmos tornaram-se constantes, apesar de serem casos raros, visto sua grande relação com diagnóstico diferencial de patologias gastrointestinais e a sua variedade de causas (SILVEIRA HJV, et al., 2012).

Os maiores representares desse grupo são os fitobezoares e os tricobezoares. Aquele, ocorre devido baixa motilidade GI em pacientes pós gastrectomia parcial de Billroth I ou II, associada a vagotomia. Esse, é caracterizado por ingestão de cabelo, mais presente em pacientes com transtornos psiquiátricos, como a tricotilomania (MENEGHELLI, UG, 2006). O acúmulo deste material ocorre, em grande número dos casos, no estômago e pode cursar com sintomas inespecíficos, o que somado a sua raridade, geralmente, dificulta a suspeita. Em estômago, o acúmulo de pelos se adapta ao formato do órgão, principalmente do antro pilórico, que pode evoluir para a Síndrome de Rapunzel, onde a formação do tricobezoar se expande até duodeno ou jejuno, sendo que na literatura atual, a maior "cauda" relatada foi de aproximadamente $110 \mathrm{~cm}$. A presença do mesmo em jejuno e íleo é raramente relatada na literatura, já que ambos órgãos não apresentam locais de estreitamente, exceto a válvula ileocecal. Comparando com a anatomia muscular e funcional gástrica, devido a contratilidade do músculo pilórico e ao estreitamento do antro gástrico, forma-se uma região favorável para surgimento de obstrução por acúmulo de cabelo (FARIA AP, et al., 2007).

Apesar dos sintomas inespecíficos, presença de dor abdominal, parada de evacuação e vômitos estão relacionados ao quadro. Irritação peritoneal, vômitos e hemorragia digestiva alta estão relacionados a casos avançados e complicações. Achados de massa em região epigástrico (sinal de Lamerton) estão associadas a tricobezoares gástricos, o que não exclui a possibilidade de encontrar massas devido a bezoares volumosos em outras topografias. Sinais de tricotilomania devem ser avaliados durante somatoscopia, já que a tricolomalácia e a tricofagia deixam encurtamento capilar irregular e áreas de alopecia (GONUGUNTLA V, JOSHI D-D, 2009).

Seu diagnóstico clínico é complicado, podendo solicitar exames de rotina para abdômen agudo, como radiografia de abdômen e tórax, além de tomografia computadorizada e ultrassonografia. No entanto, são sensíveis apenas para sinais de obstrução, e não para o diagnóstico da doença. Radiografia de abdômen revela sinais de obstrução, sendo o primeiro exame dentro da rotina de emergências abdominais, no entanto, não apresenta condições favoráveis de localização de obstrução ou a causa do mesmo. Tomografia pode ser 
mais específico neste aspecto, podendo localizar o local obstrutivo, além de revelar sinais típicos de obstrução e complicações, como dilatação de alças, pneumatose ou barramento de tecido, O único exame específico é a endoscopia digestiva alta, que avalia a presença direta de corpos estranhos em trato gastrointestinal alto. No entanto, possui suas limitações, já que, no máximo, consegue visualizar duodeno de maneira adequada (UZUNOGLU MY, et al. 2018).

O tratamento da maioria dos casos obstrutivos é cirúrgico, por enterotomia ou gastrotomia (dependendo da localização). Entretanto, o papel da endoscopia na retirada de bezoares de menor tamanho vem crescendo cada vez mais, devido a menor taxa de complicações pós-operatórias. Visando evitar complicações, deve-se acionar o acompanhamento psiquiátrico ao atendimento multidisciplinar dos casos, já que tratando a causa base, haverá menor frequência de casos recorrentes (NAIK S, et al. 2017).

O objetivo deste artigo se baseia em relatar sobre um caso de uma paciente adolescente com queixas clínicas típicas de obstrução intestinal sem causa evidente no primeiro momento, mas com o decorrer do caso, a etiologia se releva como uma patologia psiquiátrica (tricomalácia e tricofagia), além de discutir sobre o tratamento e acompanhamento pós-operatório atual.

Este relato tem a autorização de publicação da responsável pela paciente, com termo de consentimento livre e esclarecido assinado pela mesma, disponível em caso de necessidade de comprovação ética.

\section{DETALHAMENTO DO CASO}

Apresentar-se-á o caso clínico de volumosos tricobezoares intestinais identificados em um Hospital Pronto Socorro da cidade de Manaus. Trata- se de paciente do sexo feminino, 15 anos, procura o serviço de Pronto Socorro com relato de constipação intestinal há 15 dias, sem comorbidades conhecidas. Sendo realizado clister glicerinado, melhora do quadro e alta hospitalar. Após um dia, retorna ao serviço, referindo início de vômitos fecaloides, febre não aferida, dor, distensão abdominal e diurese preservada. Usou-se uma sonda nasogástrica de Levine aberta com débito de $900 \mathrm{~mL}$ de secreção amarelada fecaloide.

Ao exame físico: paciente em regular estado geral, contactuando verbalmente, abertura ocular ao estímulo verbal, hipoativa, hipocorada, desidratada, acianótica e anictérica. Segue hipotensa e bradicárdica. Abdômen globoso, ruídos hidroaéreos ausentes, flácido, doloroso a palpação profunda, sem sinais de irritação peritoneal ou presença de visceromegalias e à percussão, hipertimpanismo. Ao exame laboratorial, hiponatremia e hiposmolaridade (Sódio: 103 e potássio: 2,4). Solicitou-se tomografia computadorizada de abdômen total, evidenciando distensão difusa de todo delgado, sem fator obstrutivo aparente; conteúdo fecal em ceco, cólon ascendente e reto alto; pneumatose em pelve, sem pneumoperitônio. Sugerindo abdômen agudo obstrutivo por tricobezoar intestinal. Após avaliação pré-operatória de rotina, a paciente foi submetida à intervenção cirúrgica. Optou- se pela laparotomia exploradora com acesso mediano xifopúbico, observando distensão de alças de delgado, contendo três materiais endurecidos, posteriormente identificados e retirados como tricobezoares, entre jejuno e íleo (a $30 \mathrm{~cm}, 100 \mathrm{~cm}$ do ângulo de Treitz e a $10 \mathrm{~cm}$ da válvula íleocecal) (Figuras 1). Além disso, estômago bastante distendido com conteúdo de aspecto fecaloide aspirado por sonda nasogástrica. Sem outras alterações nos demais órgãos intracavitários.

Solicitado histopatológico dos materiais retirados na enterotomia e realizado enterorrafia em dois planos. Encaminhada a Unidade de Terapia Intensiva (UTI) sem necessidade de uso de drogas vasoativas e em ventilação espontânea. Após a intervenção, evoluiu sem intercorrências e a dieta foi retomada em 24 horas, com boa tolerância. No terceiro dia, recebeu alta da UTI e foi encaminhada para a ala cirúrgica onde seguiu hemodinamicamente estável e recebeu alta hospitalar após quatro dias de internação 
Figura 1. (A) Retirada de tricobezoar de alça intestinal com perceptível distensão das mesmas. (B) Tricobezoars retirados de intestino em mesa cirúrgica.

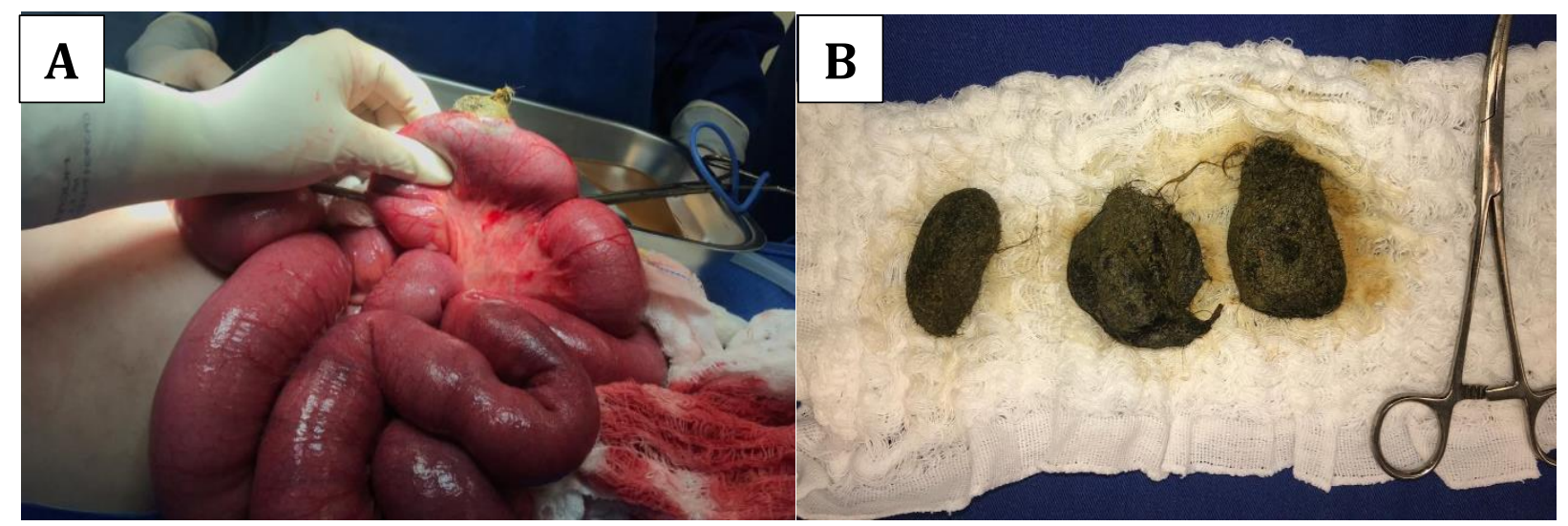

Fonte: Dados do trabalho, 2019

\section{DISCUSSÃO}

Apresentamos um caso clínico de volumosos tricobezoares intestinais identificados em uma adolescente, num serviço de Pronto Socorro (PS) na cidade de Manaus. O caso em si se encaixa dentro do grupo de síndrome de abdome agudo e síndrome obstrutiva (BRUNETTI A E SCARPELINI S, 2007)

Os tricobezoares formam-se gradual e lentamente, são originados pela ingestão de cabelos, em geral no estômago devido a sua estrutura anatômica estreita em antro (piloro), características que diferem do caso apresentado, já que o mesmo apresentou maior volume de pelos acumulados em transição jejunoileal. Como não houve formação de tricobezoares localizados em estomago, não se enquadra na Síndrome de Rapunzel, nem apresentava sintomas dispépticos, assim como alguns relatados previamente na literatura (BARGASOCHOA M, et al, 2018).

Embora raros, representam causa importante de dor abdominal e massas palpáveis, podendo ser facilmente considerado câncer em primeiro momento, devido ao diagnóstico ser complicado caso não exista suspeita para o mesmo na história patológica pregressa, problemas psiquiátricos ou eventos similares prévios. Apresenta apenas clínica de obstrução intestinal comum, como distensão abdominal, constipação e dor à palpação do abdômen, sem achados característicos em exames de imagem. Exame físico em couro cabeludo é obrigatório caso haja suspeita, podendo identificar diminuição de volume capilar ou áreas de alopecia, indicadores de tricomalacia e tricofagia (KHANNA K, et al. 2018).

Em topografia gástrica, há sintomas de plenitude pós-prandial, náuseas, vômitos, epigastralgia e, raramente, pirose. A presença de distensão abdominal e constipação estão entre as queixas mais comuns em qualquer nível do trato gastrointestinal. Relatos de complicações estão associadas ao grande volume de pelos acumulados e do tempo de permanência em alças, sendo a mais comum perfuração intestinal, marcada clinicamente pela irritação peritoneal ao exame físico do abdômen. O tratamento para o caso foi a cirúrgico, por laparotomia exploradora, enterotomia e enteroanastomose, devido a sua localização de difícil acesso por outros meios. Em realidade, devido a localização mais comum ser o estômago, muitos casos avaliam como positivo o uso de endoscopia digestiva alta (EDA) associado ou não com acesso via laparotomia, em casos de síndrome de Rapunzel, por exemplo. Há um benefício devido ao menor trauma cirúrgico e provável tempo diminuído no pós-operatório. No entanto, o caso relatado não apresentava condições favoráveis para tentativa de retirada por EDA, já que a massa obstrutiva estava localizada em porção final de jejuno e início de íleo, sendo impossível nestas situações a avaliação por imagem endoscópica (AL-OSAIL EM, et al, 2017).

REAS/EJCH | Vol.Sup.20 | e544 | DOI: https://doi.org/10.25248/reas.e544.2019 Página 4 de $\mathbf{5}$ 
Os bezoares são raros, porém o tricobezoar é a forma mais comum encontrada na população pediátrica e em meninas adolescentes com transtornos psiquiátricos. Exames de imagem, como radiografia de abdômen ou tomografia computadorizada sem contraste auxiliam o diagnóstico, mas não são específicos para a caracterização de tricobezoar, pois não existem sinais patognomonicos da doença nos exames citados, apenas sinais de obstrução intestinal e a localização da mesma. Caso o tricobezoar não seja tratado imediatamente após o diagnóstico, a taxa de mortalidade aumenta em 30\% (BRUNETTI A E SCARPELINI S, 2007).

O seguimento psicológico é indicado em todos os casos similares, assim como o psiquiátrico. Transtorno obsessivo compulsivo está associado a tricomalacia e tricofagia, e caso não seja tratado ou diminuído, recidivas do caso podem ocorrer. No entanto, a recidiva destes casos é raríssima na literatura, o que leva a concluir que o acompanhamento multidisciplinar pós-cirúrgico é eficiente. É importante incluir está patologia no diagnóstico diferencial durante avaliação de abdômen aguda em atendimento de urgências, principalmente quando há história psicossocial favorável e clínica inespecífica a outros diagnósticos (JESUS LE E NOVELLI JM, 2005).

\section{CONSIDERAÇÕES FINAIS}

Tricobezoares, apesar de raros, podem ser identificados pelo exame clínico minucioso, principalmente se atentando a presença de tricolomania. Apesar de exames de imagem serem sensíveis para localização de massas e sinais obstrutivos, os mesmos não apresentam sinais patognominicos para a doença. A remoção cirúrgica é obrigatória pelo risco de complicações fatais relacionadas (FRANCISCHI FB, et al., 2008). Na suspeita de bezoar, o diagnóstico deve ser precoce e sua conduta, imediata, a fim de evitar complicações. $O$ acompanhamento psiquiátrico é necessário para evitar novos episódios, assim como para tratar comorbidades associadas.

\section{REFERÊNCIAS}

1. AL-OSAIL EM, et al. Best management modality of trichobezoar : A case report. Int J Surg Case Rep [Internet]. Surgical Associates Ltd; 2018;53:458-60. Available from: https://doi.org/10.1016/j.ijscr.2018.11.030

2. BRUNETTI A, SCARPELINI S. Abdome Agudo. Medicina (Ribeirão Preto). 2007; 40 (3): 358-67, jul./set.

3. BARGAS-OCHOA M, et al. Síndrome de Rapunzel con doble tricobezoar simultáneo en una adolescente : Reporte de caso Rapunzel syndrome with double simultaneous trichobezoar in a teenager : Clinical Case Report. Rev Chil Pediatr. 2018;89(1):98-102.

4. FARIA AP, et al. Síndrome de Rapunzel - relato de caso : tricobezoar como causa de perfuração intestinal. $J$ Pediatr (Rio J). 2000;76:83-6.

5. FRANCISCHI FB, et al. Obstrução intestinal e tricobezoar : relato de um caso recidivante. Arq Ciênc Saúde. 2008;15(2):90-2.

6. FINALE E, et al. Rapunzel syndrome : how to orient the diagnosis. Pediatr Rep. 2018;10:37-8.

7. JESUS LE and NOVELLI JM. Tricobezoares. Rev. Col. Bras. Cir.[online]. 2005, vol.32, n.3 [cited 2019-0211], pp.157-160.

8. GONUGUNTLA V, JOSHI D-D. Rapunzel Syndrome: A Comprehensive Review of an Unusual Case of Trichobezoar. Clin Med Res. 2009;7(3):99-102.

9. KHANNA K, et al. Rapunzel syndrome : a tail too long to tell ! BMJ Case Rep. 2018;1-2.

10. KUMAR N, et al. Rapunzel syndrome in adult with mysterious presentation : a rare case report with literature review. Trop Doct. 2019;0(0):2-4.

11. MENEGHELLI, UG. Elementos para diagnóstico do Abdome Agudo. Medicina (Ribeirão Preto). 2006. 36: 283-293, abr./dez.

12. NAIK S, et al. Rapunzel syndrome reviewed and redefined. Dig Surg. 2007;24(3):157-61.

13. SILVEIRA HJV, et al. Tricobezoar gigante: relato de caso e revisão da literatura. ABCD, arq. bras. cir. dig. [online]. 2012, vol.25, n.2

14. UZUNOGLU MY, et al. Treatment results of small bowel perforations due to unusual causes. Pak J Med Sci. 2018;34(4):974-9. 